\title{
Novel n-back spatial working memory task using eye movement response
}

\author{
Cameron B. Jeter • Saumil S. Patel • Anne B. Sereno
}

Published online: 13 April 2011

(C) Psychonomic Society, Inc. 2011

\begin{abstract}
We created a novel eye movement version of the $n$-back task to measure spatial working memory (WM). Rather than one continuous trial, discrete trials were presented in order to develop a simpler WM task. In Experiment 1, we varied the visibility of the final stimulus to maximize the difference in performance between 0-back and 1-back tasks (WM effect). In Experiment 2, we administered the optimized task to children. In Experiment 3 , we further simplified the task. Both adults and children easily completed our task, displaying significant WM effects. Further, similar WM effects were obtained in our original and simplified $n$-back spatial WM tasks, demonstrating flexibility. Because WM deficits are often an early feature of disease and a marker of disease progression, our saccadic measure of spatial WM may be particularly useful in hard-to-test populations, such as patients and children, and may have application in brain-imaging studies that require discrete trials.
\end{abstract}

Keywords Voluntary saccade $\cdot$ Discrete trials $\cdot$ Neurological disease $\cdot$ Psychiatric disease

Individuals with neurological or psychiatric diseases are known to have deficits in working memory (WM) (Fleming et al., 1997; Keefe et al., 1995; McDowell \& Clementz, 1996; Park \& Holzman, 1992; Possin, Filoteo, Song, \& Salmon, 2008; van der Wee et al., 2003), the ability to temporarily store and manipulate information (Baddeley, 1992). WM deficits are often an early feature of disease and a marker of disease progression (Owen, Iddon, Hodges,

C. B. Jeter $\cdot$ S. S. Patel $\cdot$ A. B. Sereno $(\bowtie)$

Department of Neurobiology and Anatomy, University of Texas

The University of Texas Health Science Center at Houston,

6431 Fannin Street, MSB 7.160A,

Houston, 77030 TX, USA

e-mail: anne.b.sereno@uth.tmc.edu
Summers, \& Robbins, 1997; Pflueger, Gschwandtner, Stieglitz, \& Riecher-Rössler, 2007; Sartori \& Edan, 2006). Thus, quantitative measurement of WM ability aids disease assessment and appropriate treatment selection. Currently, measures of spatial WM (memory for locations) in normal adults are estimated using a variety of methods. These include pen-and-paper tests (e.g., dot test; Keefe et al., 1995), computerized tests requiring a manual response such as a buttonpress or screen touch [e.g., the delayed response task (Luciana, Depue, Arbisi, \& Leon, 1992), $n$-back task (Callicott et al., 1998), or vibrotactile $n$-back task (Klatzky et al., 2008)], and eye movement tasks [e.g., the delayed prosaccade task (Hutton, Joyce, Barnes, \& Kennard, 2002; Peltsch, Hoffman, Armstrong, Pari, \& Munoz, 2008) or sequential memory task (Baumann, Frank, Rutschmann, \& Greenlee, 2007; Hutton et al., 2002; Parton et al., 2007; Peltsch et al., 2008)].

Current measures of spatial WM are effective, yet problems arise when measurements are required from patients and children. First, many neurological and psychiatric patients can have perceptual deficits impairing their ability to distinguish, for example, the colors and shapes utilized in many spatial WM tasks (Melun, Morin, Muise, \& DesRosiers, 2001; Pieri, Diederich, Raman, \& Goetz, 2000). Additionally, neurological and psychiatric patient populations demonstrate slowing in the initiation and execution of manual responses (e.g., keypresses) relative to healthy participants (Benson, 1990; Bermanzohn \& Siris, 1992; Hardy \& Hinkin, 2002; Jogems-Kosterman, Zitman, Van Hoof, \& Hulstijn, 2001; Rosofsky, Levin, \& Holzman, 1982; Sobin \& Sackeim, 1997). Psychomotor slowing is specific to manual response tasks but not to eye movement tasks (Gale \& Holzman, 2000; Reuter \& Kathmann, 2004) and frequently complicates comparisons of WM function in patients and healthy controls, since longer mean response 
times lead to baseline differences and larger variances of the data (Wagenmakers \& Brown, 2007; Wagenmakers, Grasman, \& Molenaar, 2005). Indeed, many clinical rating scales assess the motor abnormalities of patient populations such as Parkinson disease and Tourette syndrome. Despite their abnormal body movements, patients with these disorders are known to have normal reflexive saccadic eye movements (Briand, Strallow, Hening, Poizner, \& Sereno, 1999; Jeter, Patel, Butler, \& Sereno, 2009). Because normal reflexive saccadic eye movements indicate intact visual sensory and final common brain-stem motor processes, concomitant abnormal voluntary eye movements can indicate specificity for impaired cognitive functions. This suggests that WM tasks based on eye movements should have significant usefulness, compared to body motor responses, in evaluating WM in a variety of patient populations with additional perceptual and/or motor slowing deficits. Further advantages of eye movement tasks for measuring spatial WM include quick administration and easy-to-follow instructions for all ages and cognitive abilities.

In previous eye movement studies of WM, initial gain error (under- or overshooting the first target) would cause the entire trial to abort (Hodgson, Dittrich, Henderson, \& Kennard, 1999; Parton et al., 2007), hence requiring longer testing sessions to measure WM deficits in patient and child populations that are also more prone to fatigue and distraction. Further, these tasks do not allow for careful and easy manipulation of WM requirements (WM load) and task difficulty. Such manipulations are important for WM assessment in a wide variety of special populations, ranging from young children to aging adults.

A simple, quickly administered, yet sensitive spatial WM task is needed for hard-to-test populations. Thus, we created a variant of the spatial $n$-back task (Nagel, Ohannessian, \& Cummins, 2007; van der Wee et al., 2003) for use with an infrared eyetracker. In the classic spatial $n$-back task, participants respond when the current stimulus's location matches the location of the one presented $n$ items ago. Our $n$-back spatial WM task is composed of a set of discrete trials, rather than the single continuous trial used traditionally, and so allows for brief pauses that are desirable for hard-to-test populations. Each trial in our $n$-back spatial WM task uses a sequence of visual stimuli. The length of the sequence is varied from trial to trial, so that the participant must remember and update the stimulus to be remembered until the stimulus sequence has ended and a go signal has occurred. Each stimulus in the sequence is presented at one of several possible locations in the visual periphery while the participant is fixating a central visual cue. Participants respond, after the stimulus sequence has finished and a go signal has occurred, by looking at the location of the stimulus $n$ items before the final stimulus. This design offers an easily scalable task (i.e., selectable sequence lengths, number of possible stimulus locations, and $n$ in the $n$-back requirement) that might be effective at various levels of difficulty and yet permit a precise quantitative measure of WM function.

\section{Overview of experiments}

The primary goal of this research was to develop a variant of the spatial $n$-back task for use with eye movement responses. For all experiments, we tested participants on two levels of $n, 0$ and 1 . We expected that participants would have more difficulty in the 1-back blocks than in the 0-back blocks, because the former requires a higher WM load. That is, in the 0 -back blocks, the participant need only remember the most recent stimulus location and replace the contents of WM (i.e., update WM) with each subsequent stimulus presentation, whereas in the 1-back blocks, he or she must remember the last two stimuli and update these locations as each additional stimulus appears. In this way, all sensory and motor demands of the two conditions remained the same, and any differences in performance could be attributed to this additional WM load. We used the error rates and response times of saccadic eye movements as the performance measures in our 0-back and 1-back tasks. For each of these performance measures, we defined the WM effect as the difference between their values in the 1-back and 0-back tasks (1-back - 0-back).

In Experiment 1, we tested how a transient versus a remaining final stimulus influenced the WM effect in our $n$ back spatial WM task. We found that the $n$-back spatial WM task with a transient final stimulus produced a larger difference in eye movement performance between the 1back and 0-back tasks (i.e., a larger WM effect). In the next two experiments, we therefore exclusively utilized the transient final stimulus. Experiment 2 tested the utility of the $n$-back spatial WM task in children, and in Experiment 3 we tested the effectiveness of a simplified version of the task (shortened sequence lengths and fewer possible stimulus locations).

\section{Experiment 1}

The manipulations in Experiment 1 were made primarily to optimize task design. We hypothesized that the enduring presence of a final stimulus in the 1-back blocks could serve as an external cue (i.e., where not to look), decreasing error rates and response times, as has been demonstrated in other spatial memory tasks (e.g., Fitting, Wedell, \& Allen, 2007). This would make WM load lighter, thus decreasing the difficulty in this condition relative to the 0 -back blocks. 
If this hypothesis is true, it would undesirably reduce the sensitivity of our task for detecting changes in WM across the 0 - and 1-back blocks. Given that we are trying to maximize our sensitivity to WM changes across the two blocks, we would thus avoid this design. On the other hand, it is possible that the final stimulus's presence could serve as a greater distraction, increasing error rates and response times (see, e.g., Corneil \& Munoz, 1996; Toepper et al., 2010) in the 1-back blocks, and thus increase the difficulty of this condition relative to the 0 back blocks. In this scenario, the final stimulus's presence would desirably enhance our sensitivity to WM changes across the two blocks.

\section{Method}

Participants Fourteen healthy adults (20-27 years of age, mean $24.4 \pm 2.3$ years; 6 male, 8 female) were recruited from the community by flyers. The University of Texas Health Science Center at Houston Committee for the Protection of Human Subjects approved all experiments (Exps. 1, 2, and 3), which were conducted in accordance with the Declaration of Helsinki, and adult participants (and guardians of child participants) gave informed consent before participating. Children also gave assent.

Apparatus The stimuli were presented on a monitor (LCD, 17 in., $75-\mathrm{Hz}$ refresh rate, $1,024 \times 768$ pixels) connected to a Power G4 Macintosh computer running the OS 9 operating system. An infrared eyetracking camera (ISCAN ETL-200, Burlington, MA) measured eye movements. The eyetracker was connected to the Macintosh via a USB port. A custom program developed using commercially available software (Vision Shell and Code Warrior) was used to present visual stimuli and record eye movements at $240 \mathrm{~Hz}$. For each trial in all experiments, the eye position data were analyzed online, automatically canceling trials on which participants broke fixation early or blinked, and later representing these trials in random order.

Procedure Each participant was seated $72 \mathrm{~cm}$ from the center of a computer screen in a dark room with his or her head held in place by a chin-and-forehead rest. Participants were instructed to visually fixate on a $0.2^{\circ}$-diameter central circle (see Fig. 1a). Each trial sequence had a variable number of stimuli, which is crucial for maintaining uncertainty about which stimulus will become the target, thus forcing participants to remember and update the stimuli as they are presented. After $880 \mathrm{~ms}$ of fixation, three or four stimuli appeared in sequence (without repeating a location), each within one of six landmark boxes $\left(1.1^{\circ} \times 1.1^{\circ}\right)$ evenly distributed in a circle at $5.6^{\circ}$ eccentricities around the fixation point (see Fig. 1a). The stimuli $\left(0.2^{\circ} \times 0.2^{\circ}\right)$ appeared for $200 \mathrm{~ms}$ each with an interstimulus interval (ISI) of $200 \mathrm{~ms}$. Following presentation of the final stimulus, a 500-ms delay (delay period) occurred before the fixation point disappeared.

Participants completed two WM conditions (0-back and 1-back) for each of two final-stimulus conditions (visible and not visible), creating four blocks of 120 trials each. Block order was counterbalanced. In the 0-back blocks, participants were instructed to look, after fixation disap-

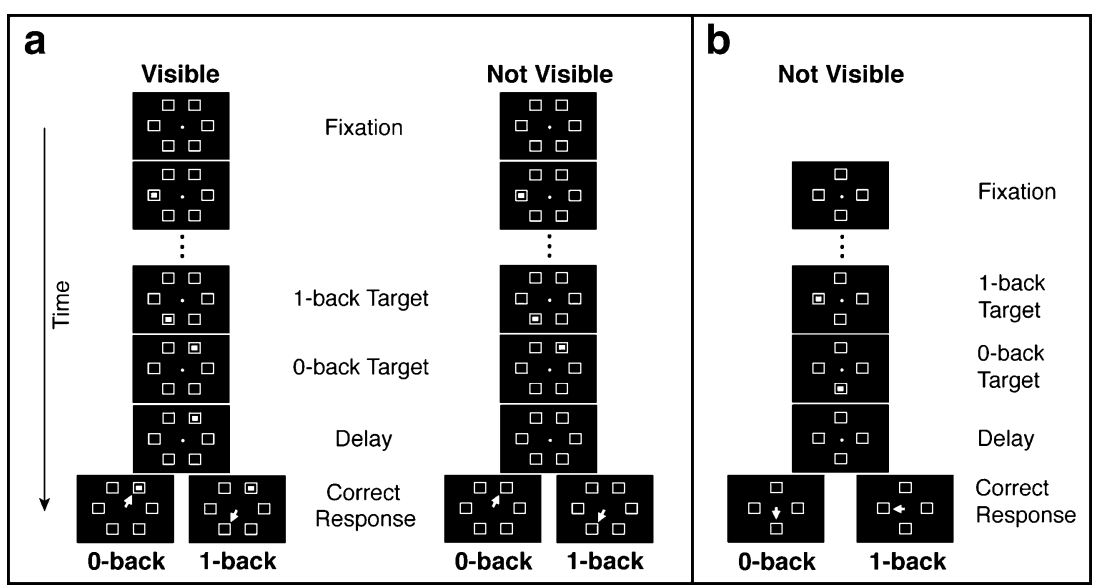

Fig. 1 Novel eye movement-based $n$-back spatial working memory tasks. (a, left) Visible condition (Exp. 1): Blocks in which the final stimulus remained visible. A trial began when fixation was maintained. Three or four sequential stimuli appeared in the six open boxes, without location repeats (three shown here). After a delay, an eye movement response (depicted by the white arrow) was made to the 0-back (last) or the 1-back (second-to-last) target, depending on the trial block. (a, right) Not-visible condition (Exps. 1 and 2): Blocks in which the final stimulus disappeared (cf. the delay and response periods of the visible and not-visible conditions). The trial sequence was the same as in the visible condition. (b) Experiment 3, with a simplified design and transient final stimulus. Once fixation was maintained, two or three sequential stimuli appeared in the four peripheral boxes without repeating a location (two shown here). After the delay, an eye movement response was made to the 0-back (last) or 1-back (second-to-last) target in different blocks 
pearance, at the location of the last stimulus in the sequence, whereas in the 1-back blocks they were told to look at the second-to-last stimulus. Eye movements were counted as correct if the initial saccade landed in the correct stimulus's circular target window ( $2^{\circ}$ radius), centered on each landmark box. In the visible blocks, the final stimulus in the sequence remained visible throughout the trial (including the delay period, disappearance of the fixation point, and the response period), whereas in the not-visible blocks it vanished from the screen after a 200-ms presentation (before the delay period).

Statistical analyses Trials with a response time less than $100 \mathrm{~ms}$ or greater than $900 \mathrm{~ms}$ were discarded. Averaged across all experiments, $12.8 \%$ of the 0 -back trials and $12.5 \%$ of the 1 -back trials were discarded. From the remaining trials, the error rate was calculated as the percentage of trials on which the participant made an eye movement to an incorrect location. Correct trials were further trimmed if the response time was 2.5 standard deviations from the participant's mean. This procedure eliminated $7.7 \%$ of the original data, averaged across all experiments. Mean response times were calculated from these remaining trials. Repeated measures ANOVAs were conducted with the factors WM condition (0- and 1-back) and, for Experiment 1 only, final-stimulus condition (visible and not visible), with error rate and response time as dependent measures. Planned contrasts were used to compare WM condition in all Experiments. For Experiment 1 only, we also performed a planned paired $t$ test to determine whether the WM effect differed in the visible and not-visible conditions, using the mean squared error of the interaction term from the ANOVA. There were no speedaccuracy trade-offs in any of the experiments. Because there were very small error rates in the visible and not-visible 0 -back conditions of Experiment $1(1.44 \%$ and $0.90 \%$, respectively) and Kolmogorov-Smirnov and Shapiro-Wilk tests showed these error rates to be nonnormally distributed, we additionally performed a nonparametric Wilcoxon signed rank test on these error data. We report the results of both the $t$ and Wilcoxon tests in the text, and only the Wilcoxon results in Fig. 2.

\section{Results}

As seen in Fig. 2a, left side, when the final stimulus was visible, participants showed a WM effect (1-back minus 0back) for error rates, with significantly more errors in the 1back (solid bars) WM condition than in the 0-back (open bars) WM condition $[6.99 \%$ vs. $1.44 \% ; F(1,13)=60.80$, $p<.001$, and $Z=-3.04, p<.01]$. Response times were not significantly different between the 1-back and 0-back WM conditions $[253$ vs. $241 \mathrm{~ms} ; F(1,13)=2.14, p>.17$; Fig. 2b, left side]. When the final stimulus was not visible, participants also showed a significant WM effect for error rates $[8.73 \%$ vs. $0.90 \% ; F(1,13)=121.05, p<.001$, and $Z=-3.30, p<.001$; Fig. 2a, right side], but, in addition, displayed a response time difference between the conditions [277 vs. $252 \mathrm{~ms} ; F(1,13)=10.09, p<.01$; Fig. $2 \mathrm{~b}$, right side]. Furthermore, a comparison of WM effects by finalstimulus condition showed that this WM effect for error rates when the final stimulus was not visible was significantly greater than the WM effect for error rates in the condition when the final stimulus was visible $[7.84 \%$ vs. $5.55 \% ; F(1,13)=10.27, p<.01]$. The Wilcoxon signed rank test also was significant $(Z=-2.10, p<.05)$.

Incorrect eye movements in the 1-back condition were directed to the last stimulus in the sequence more than to any other position (Fig. 3). Interestingly, significantly more incorrect eye movements were directed to the position of the last stimulus in the sequence in the not-visible condition than in the visible condition $[3.78$ vs. $0.88 \% ; F(1,16.8)=$ $11.20, p<.01]$.

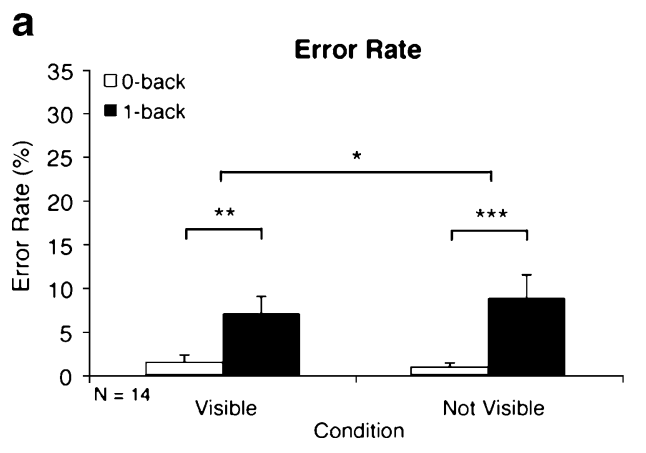

Fig. 2 Working memory effects in adults (Exp. 1). Error rate (a) and response time (b) WM effects are illustrated by significant differences between the 1-back (solid bars) and 0-back (open bars) blocks, shown b

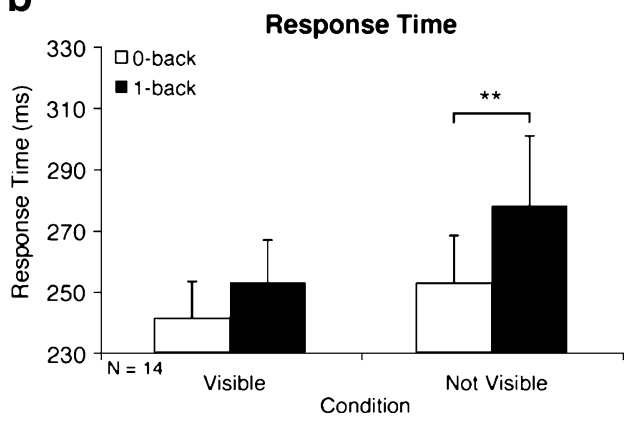

separately for the visible and not-visible conditions. Error bars are standard errors of the means. For error rates, significance is indicated from Wilcoxon signed rank tests. ${ }^{*} p<.05 .{ }^{* *} p<.01 .{ }^{* * * *} p<.001$ 


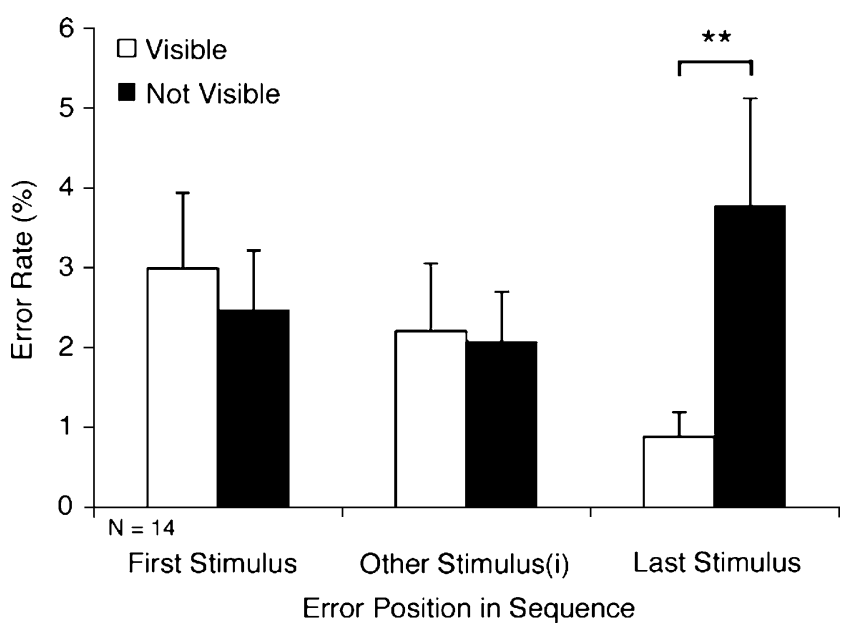

Fig. 3 Positions of eye movement errors in the 1-back condition (Exp. 1) when the sequence consisted of three or four stimuli. Thus, in a three-stimulus trial, the middle bars represent errors to the second stimulus, whereas in a four-stimulus trial they represent the average of the error rates to the second and third stimuli. Denotation of significance level is the same as in Fig. 2

\section{Discussion}

Experiment 1 ascertained (1) whether our simple designs, both visible and not visible, would yield an effective measure of WM load and (2) whether a visible (remaining) or not visible (transient) final stimulus resulted in the largest WM effect. Whereas both visible and not-visible designs produced a significant WM effect, it was the not-visible condition that produced a significant WM effect for response times as well as a greater significant WM effect for error rates than the visible condition. The fewer errors to the location of the last stimulus in the sequence in the visible 1-back condition than in the notvisible 1-back condition suggest that a visible final stimulus served as an external cue (i.e., where not to look), decreasing error rates to the final stimulus location and decreasing response times to the proper 1-back stimulus location (see Fig. 2). Thus, in the 1-back blocks, a visible or remaining final target undesirably served as a cue and slightly, but significantly, diminished the task condition's ability to measure spatial WM. We therefore exclusively used a not- visible design in subsequent experiments. In Experiment 2, we determined whether our novel $n$-back spatial WM task could measure WM load in children.

\section{Experiment 2}

\section{Method}

Participants Eleven healthy children (8-16 years of age, mean $12.2 \pm 2.7$ years; 7 male, 4 female) were recruited from the community by flyers.

Procedure Children completed 0-back and 1-back blocks of our $n$-back spatial WM task with a transient final stimulus (Fig. 1a, not-visible sequence; maximum length of the sequence $=4$, number of possible locations $=6$ ). The task procedure was identical to that in Experiment 1.

\section{Results}

The WM effect was significant for error rates [25.81\% vs. $4.25 \% ; F(1,10)=6.58, p<.05]$ but was not significant for response times $[281$ vs. $276 \mathrm{~ms} ; F(1,10)=0.07, p>.80$; see Fig. $4 \mathrm{a}$ and $\mathrm{b}$, respectively]. A repeated measures ANOVA with WM Condition (0- or 1-back) as a withinsubjects factor and Experiment (1 or 2) as the betweensubjects factor showed that compared to the adults in Experiment 1, children had a significantly greater WM effect for error rates $[7.84 \%$ vs. $21.56 \% ; F(1,23)=5.86$, $p<.05]$ but a comparable WM effect for response times [25 vs. $5 \mathrm{~ms} ; F(1,23)=1.39, p>.25]$.

\section{Discussion}

Experiment 2 demonstrated that children could easily complete our $n$-back spatial WM task. Further, like adults, children showed a significant WM effect for error rates. This greater effect in children than in adults corroborates WM developmental differences described in the literature (Swanson, 1999;
Fig. 4 Working memory effects in children (Exp. 2). Error rate (a) and response time (b) WM effects are illustrated by significant differences between 1-back (solid bars) and 0-back (open bars) blocks. Denotation of significance level is the same as in Fig. 2 a

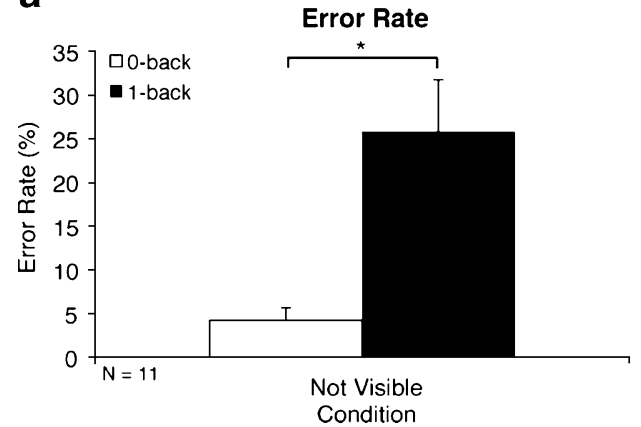

b

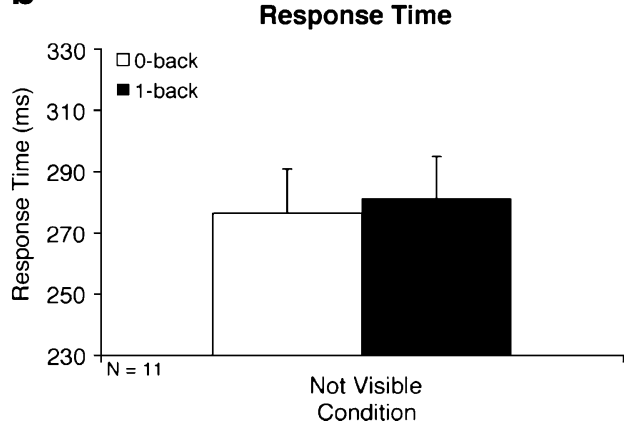


Thomason et al., 2009). To test whether our tasks are robust in detecting WM effects even with a simpler (shorter and scaled) design that might be needed for younger children or patient populations, in Experiment 3 we reduced the number of trials, sequence lengths, and possible stimulus locations.

\section{Experiment 3}

Method

Participants We tested 16 healthy adults (20-29 years of age, mean $24.6 \pm 2.8 ; 5$ male, 11 female), who were recruited from the community by flyers, on this scaled version of our WM tasks (Fig. 1b; maximum length of the sequence $=3$, number of possible locations $=4$ ).

Procedure After $750 \mathrm{~ms}$ of fixation, a sequence of just two or three stimuli at $7^{\circ}$ eccentricities appeared (without repeats) only in the four cardinal locations around the fixation point. Stimuli appeared for $80 \mathrm{~ms}$ with an ISI of $350 \mathrm{~ms}$; again, the final stimulus was not visible. In separate, counterbalanced 96-trial blocks, participants were told to look to the 0-back (last) stimulus or the 1-back (second-to-last) stimulus.

\section{Results}

The WM effect was significant for error rates $[6.88 \%$ vs. $2.14 \% ; F(1,15)=11.28, p<.01]$, but not for response times $[308$ vs. $313 \mathrm{~ms} ; F(1,15)=0.11, p>.74$; Fig. $5 \mathrm{a}$ and $\mathrm{b}$, respectively]. Finally, whereas Experiment 3 was a scaled version of Experiment 1, a repeated measures ANOVA with WM Condition (0- or 1-back) as a withinsubjects factor and Experiment (1 or 3 ) as the betweensubjects factor found that both experiments produced WM effects of similar magnitudes [error rate WM effect, $4.74 \%$ vs. $7.84 \%$, respectively; $F(1,28)=1.33, p>.26$; response time $\mathrm{WM}$ effect, -5 vs. $25 \mathrm{~ms}$, respectively; $F(1,28)=$ 2.67, $p>.11]$.
Discussion

Experiment 3 showed that a simplified version of our novel $n$-back spatial WM task was an effective measure of WM load. Reducing the number of trials, sequence lengths, and possible stimulus locations considerably shortened the duration of the trial blocks while maintaining task effectiveness. Additionally, our simplified version generated WM effects comparable to those in our original $n$-back spatial WM task.

\section{General discussion}

Taken together, our findings show that our novel and simple eye movement task is able to quickly and robustly measure spatial WM in adults and children. Consistent with prior literature (Swanson, 1999; Thomason et al., 2009), this task is also sensitive enough to show that children have reduced WM ability (as evidenced by a greater WM effect for error rates) as compared to adults. We demonstrated that a transient (as opposed to remaining) final target elicited a greater WM effect. Finally, we demonstrated that a further simplified and shortened task produces WM effects similar to those from the original design.

The ability of our task to yield similar WM effects under different spatial configurations suggests that our design is flexible and robust. These properties allow for appropriate customization of task difficulty (including increasing the trial sequence length or the load to 2-back, etc.), and suggest that such a task enables choice of task difficulty while maintaining task sensitivity.

Our novel spatial WM task retains elements of the classic spatial $n$-back task essential for measuring WM, yet it incorporates an important primary difference: Namely, where stimuli are continuously presented in the classic spatial $n$-back task, our task partitions the stimuli into discrete trials containing just a few, variable number of stimuli. Thus, the participant needs to simultaneously read and write stimuli into WM only one or two times per trial in
Fig. 5 Working memory effects in a simplified design (Exp. 3). Error rate (a) and response time (b) WM effects are illustrated by significant differences between 1-back (solid bars) and 0-back (open bars) blocks. Denotation of significance level is the same as in Fig. 2 a

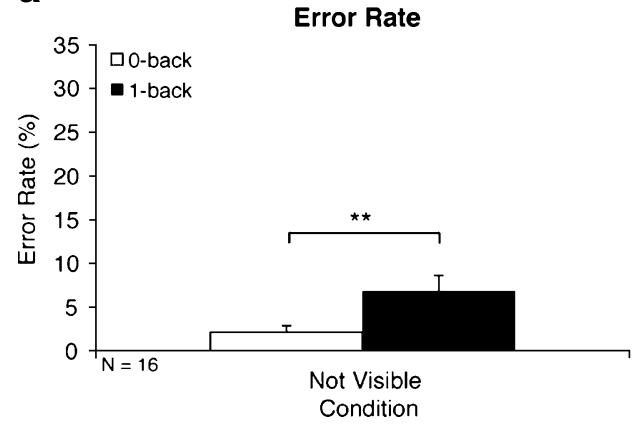

b

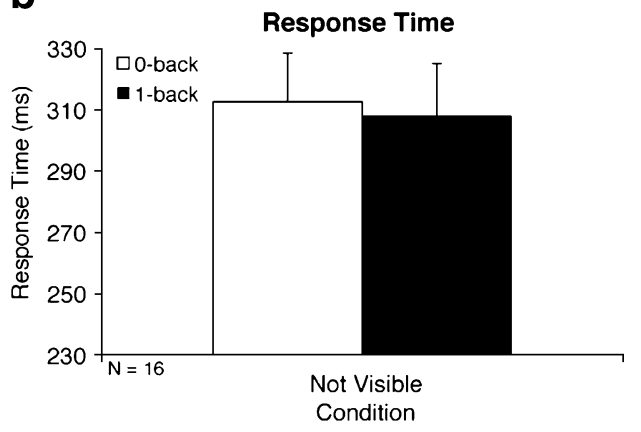


our 1-back condition, as opposed to continuously and repeatedly with each new target in the classic $n$-back task. This design difference allows optimal adaptation of the task for hard-to-test populations.

Past studies of WM have also employed $n$-back tasks with few stimulus locations and continuously present landmarks. Callicott et al. (1998) administered an $n$-back task with four stimulus locations and continuously present landmarks to schizophrenic patients. Likewise, we designed our simple task with hard-to-test populations in mind. Our novel $n$-back task, like memory-guided saccade and delayed matching-to-sample tasks, can be designed to include a larger set of stimulus positions. This is one of the advantageous, scalable parameters of our task, in addition to the flexibility of allowing longer sequences and longer delays.

Because our task used a limited number of locations and did not employ an articulatory suppression task, it is possible that participants used verbal strategies. Verbal labels can be an integral part of WM, specifically maintenance and retrieval. Verbal rehearsal has been shown to help recall of intermediate stimuli (rather than first or last stimuli) in a list memory task (in which stimuli are presented in a sequence, similar to the $n$-back task; Wright et al., 1990).

Importantly, our short ISIs (200 or $350 \mathrm{~ms}$ ) hamper the ability of participants to use a verbal strategy. Increased accuracy with longer ISIs in a list memory task indicates a positive effect of rehearsal on WM performance (Intraub, 1980; Proctor, 1983). In these studies, performance was equivalent regardless of whether the stimuli were presented for $5 \mathrm{~s}$ with no ISI or for $110 \mathrm{~ms}$ with a 4,890-ms ISI. Note that a brief stimulus $(110 \mathrm{~ms})$ was encoded. Performance decreased only with decreasing ISI (less time for rehearsal). Thus, the short ISI in our task gives little time to rehearse, rendering the effect of verbal rehearsal or labeling minimal. Finally, it has been argued that only object WM depends on verbal mediation (Simons, 1996) and that there is greater sensitivity of object than of spatial WM performance to manipulations of verbal processing (Postle, D'Esposito, \& Corkin, 2005). In support of these studies, Vuontela, Rama, Raninen, Aronen, and Carlson (1999) used a spatial $n$-back task without articulatory suppression and asked participants what strategies they used in the task. Of those reporting a strategy, the majority of the participants used a spatial, nonverbal strategy.

Our WM study is not unique in using brief stimulus durations, as others have used $80 \mathrm{~ms}$ (Wright et al., 1990), 100 ms (Vogel, Woodman, \& Luck, 2006; Vuontela et al., 1999), or $110 \mathrm{~ms}$ (Intraub, 1980). Further, change detection tasks probe memory with brief presentations of an array of stimuli. Our stimulus durations ( 80 or $200 \mathrm{~ms}$ ) are in the same range in which other participants have achieved encoding and maintenance (Luck \& Vogel, 1997; Sperling, 1963). Moreover, in these tasks, participants had to encode not one, but many, stimuli. Luck and Vogel showed that there was no performance difference between arrays presented for 100 and $500 \mathrm{~ms}$ (but, see also, Bays, Catalao, \& Husain, 2009).

In the case of ISI durations, consolidation of stimuli into durable WM representations has historically been estimated to be a slow process $(500 \mathrm{~ms})$. Vogel et al. (2006), however, measured the time course of consolidation as being closer to $50 \mathrm{~ms}$ per stimulus. Our shortest ISI was several times longer than this estimate $(200 \mathrm{~ms})$.

Our novel measure of WM function eliminates the pitfalls of prior WM task designs. Our task does not require discrimination of colors or shapes, which may be affected or compromised in different patient populations, and it is rapid enough for hard-to-test populations, which have problems maintaining attention for an extended period of time. Use of an eye movement response often eliminates baseline differences inherent in patient populations who have motor slowing (Gale \& Holzman, 2000; Hardy \& Hinkin, 2002; Reuter \& Kathmann, 2004; Rosofsky et al., 1982; Sobin \& Sackeim, 1997). Further, using the 0-back WM condition as a baseline allows for a more direct and "pure" measure of the effect of increasing WM load.

Many eye-movement-based tasks for measuring WM have drawbacks. In delayed or remembered prosaccade tasks, delay durations must be increased to seconds to get measurable memory effects, increasing not only trial durations but also trial failures, and making tasks burdensome (Amador, Hood, Schiess, Izor, \& Sereno, 2006). Further, to retain reasonable testing session durations with these longer trials, the number of trials collected in a session must be reduced, undesirably diminishing the statistical power. In contrast, our task successfully measures WM effects for multiple locations over varied time periods, including quite short delays. Sequential memory tasks often require memory storage of, and eye movements to, each of multiple stimulus locations. Also, in previous sequential eye movement task designs, gain errors during the initial eye movement caused the entire trial to abort (Hodgson et al., 1999; Parton et al., 2007). Landmarks (open boxes, Fig. 1) at our possible stimulus locations improve eye movement spatial accuracy and avoid trial termination. We are currently utilizing this task in various neurological patient populations and are able to show increased WM deficits in patients with Tourette syndrome, attention deficit hyperactivity disorder, and obsessive compulsive disorder, relative to healthy participants (Jeter et al., 2009).

Our task can be adapted easily for fMRI mixed block/ event-related design studies and has advantages over traditional, continuous $n$-back fMRI tasks (e.g., Buckner et al., 1998; Visscher et al., 2003). Our single-trial design 
allows for breaks between the end of one trial and the beginning of the next. This facilitates temporal synchronization with other external events (e.g., the start of a scan) and prevents overlap of the hemodynamic responses to one trial's response with the next trial's onset, which is a common problem in continuous $n$-back tasks within an fMRI blocked design (Nagel et al., 2007). Further, using discrete trials allows for the onsets of trials to be jittered (variable delay between trials), preventing a correlated structure of trial onset and offset and increasing statistical efficiency (Dale, 1999). The jittering also creates temporal uncertainty, thus helping participants maintain their attention on the task.

In summary, our task is suitable for all ages and is appropriate for clinical populations. The design is scalable for difficulty, producing robust results regardless of the sequence length or possible stimulus locations (cf. Exps. 1 and 3). Additionally, our test does not require many participants per comparison group, since the effect size is large enough to convey power to detect small WM differences. Further, the task could also be used for monkey behavioral and physiological studies, to address the underlying neural mechanisms of spatial WM processing. Perhaps most directly, application of this task in patient populations to detect the onset or progression of WM deficits may be critical in providing assessment and evaluation of treatment effects on WM.

Acknowledgement The study was funded by grants from the NIH (P30-EY010608; CTSA Grant TL1RR024147) and the NSF (0924636). The authors thank Emily Smitherman and Jennifer Dulin for help with data collection, Anthony Wright and Tim Ellmore for comments on earlier drafts, and Alice Chuang for help with statistical analyses.

\section{References}

Amador, S. C., Hood, A. J., Schiess, M. C., Izor, R., \& Sereno, A. B. (2006). Dissociating cognitive deficits involved in voluntary eye movement dysfunctions in Parkinson's disease patients. Neuropsychologia, 44, 1475-1482.

Baddeley, A. (1992). Working memory. Science, 255, 556-559.

Baumann, O., Frank, G., Rutschmann, R. M., \& Greenlee, M. W. (2007). Cortical activation during sequences of memory-guided saccades: A functional MRI study. Neuroreport, 18, 451-455.

Bays, P. M., Catalao, R. F., \& Husain, M. (2009). The precision of visual working memory is set by allocation of a shared resource. Journal of Vision, 9, 1-11.

Benson, F. (1990). Psychomotor retardation. Neuropsychiatry, Neuropsychology, and Behavioral Neurology, 3, 36-47.

Bermanzohn, P. C., \& Siris, S. G. (1992). Akinesia: A syndrome common to parkinsonism, retarded depression, and negative symptoms of schizophrenia. Comprehensive Psychiatry, 33, 221-232.

Briand, K. A., Strallow, D., Hening, W., Poizner, H., \& Sereno, A. B. (1999). Control of voluntary and reflexive saccades in Parkinson's disease. Experimental Brain Research, 129, 38-48.
Buckner, R. L., Koutstaal, W., Schacter, D. L., Dale, A. M., Rotte, M., \& Rosen, B. R. (1998). Functional-anatomic study of episodic retrieval: II. Selective averaging of event-related fMRI trials to test the retrieval success hypothesis. Neuroimage, 7, 163-175.

Callicott, J. H., Ramsey, N. F., Tallent, K., Bertolino, A., Knable, M. B., Coppola, R., et al. (1998). Functional magnetic resonance imaging brain mapping in psychiatry: Methodological issues illustrated in a study of working memory in schizophrenia. Neuropsychopharmacology, 18, 186-196.

Corneil, B. D., \& Munoz, D. P. (1996). The influence of auditory and visual distractors on human orienting gaze shifts. The Journal of Neuroscience, 16, 8193-8207.

Dale, A. M. (1999). Optimal experimental design for event-related fMRI. Human Brain Mapping, 8, 109-114.

Fitting, S., Wedell, D. H., \& Allen, G. L. (2007). Memory for spatial location: Cue effects as a function of field rotation. Memory \& Cognition, 35, 1641-1658.

Fleming, K., Goldberg, T. E., Binks, S., Randolph, C., Gold, J. M., \& Weinberger, D. R. (1997). Visuospatial working memory in patients with schizophrenia. Biological Psychiatry, 41, 43-49.

Gale, H. J., \& Holzman, P. S. (2000). A new look at reaction time in schizophrenia. Schizophrenia Research, 46, 149-165.

Hardy, D. J., \& Hinkin, C. H. (2002). Reaction time performance in adults with HIV/AIDS. Journal of Clinical and Experimental Neuropsychology, 24, 912-929.

Hodgson, T. L., Dittrich, W. H., Henderson, L., \& Kennard, C. (1999). Eye movements and spatial working memory in Parkinson's disease. Neuropsychologia, 37, 927-938.

Hutton, S. B., Joyce, E. M., Barnes, T. R., \& Kennard, C. (2002). Saccadic distractibility in first-episode schizophrenia. Neuropsychologia, 40, $1729-1736$.

Intraub, H. (1980). Presentation rate and the representation of briefly glimpsed pictures in memory. Journal of Experimental Psychology: Human Learning and Memory, 6, 1-12.

Jeter, C. B., Patel, S. S., Butler, I. J., \& Sereno, A. B. (2009). Saccadic measures of inhibitory control and working memory in Tourette syndrome subtypes (Program No. 575.24) [Abstract]. 2009 Neuroscience Meeting Planner. Washington, DC: Society for Neuroscience.

Jogems-Kosterman, B. J., Zitman, F. G., Van Hoof, J. J., \& Hulstijn, W. (2001). Psychomotor slowing and planning deficits in schizophrenia. Schizophrenia Research, 48, 317-333.

Keefe, R. S., Roitman, S. E., Harvey, P. D., Blum, C. S., DuPre, R. L., Prieto, D. M., et al. (1995). A pen-and-paper human analogue of a monkey prefrontal cortex activation task: Spatial working memory in patients with schizophrenia. Schizophrenia Research, $17,25-33$.

Klatzky, R. L., Giudice, N. A., Marston, J. R., Tietz, J., Golledge, R. G., \& Loomis, J. M. (2008). An $n$-back task using vibrotactile stimulation with comparison to an auditory analogue. Behavior Research Methods, 40, 367-372.

Luciana, M., Depue, R. A., Arbisi, P., \& Leon, A. (1992). Facilitation of working memory in humans by a D2 dopamine receptor agonist. Journal of Cognitive Neuroscience, 4, 58-68.

Luck, S. J., \& Vogel, E. K. (1997). The capacity of visual working memory for features and conjunctions. Nature, 390, 279-281.

McDowell, J. E., \& Clementz, B. A. (1996). Ocular-motor delayedresponse task performance among schizophrenia patients. Neuropsychobiology, 34, 67-71.

Melun, J. P., Morin, L. M., Muise, J. G., \& DesRosiers, M. (2001). Color vision deficiencies in Gilles de la Tourette syndrome. Journal of the Neurological Sciences, 186, 107-110.

Nagel, B. J., Ohannessian, A., \& Cummins, K. (2007). Performance dissociation during verbal and spatial working memory tasks. Perceptual and Motor Skills, 105, 243-250. 
Owen, A. M., Iddon, J. L., Hodges, J. R., Summers, B. A., \& Robbins, T. W. (1997). Spatial and non-spatial working memory at different stages of Parkinson's disease. Neuropsychologia, 35, 519-532.

Park, S., \& Holzman, P. S. (1992). Schizophrenics show spatial working memory deficits. Archives of General Psychiatry, 49, 975-982.

Parton, A., Nachev, P., Hodgson, T. L., Mort, D., Thomas, D., Ordidge, R., et al. (2007). Role of the human supplementary eye field in the control of saccadic eye movements. Neuropsychologia, $45,997-1008$.

Peltsch, A., Hoffman, A., Armstrong, I., Pari, G., \& Munoz, D. P. (2008). Saccadic impairments in Huntington's disease. Experimental Brain Research, 186, 457-469.

Pflueger, M. O., Gschwandtner, U., Stieglitz, R. D., \& RiecherRössler, A. (2007). Neuropsychological deficits in individuals with an at risk mental state for psychosis - Working memory as a potential trait marker. Schizophrenia Research, 97, 14-24.

Pieri, V., Diederich, N. J., Raman, R., \& Goetz, C. G. (2000). Decreased color discrimination and contrast sensitivity in Parkinson's disease. Journal of the Neurological Sciences, 172, $7-11$.

Possin, K. L., Filoteo, J. V., Song, D. D., \& Salmon, D. P. (2008). Spatial and object working memory deficits in Parkinson's disease are due to impairment in different underlying processes. Neuropsychology, 22, 585-595.

Postle, B. R., D’Esposito, M., \& Corkin, S. (2005). Effects of verbal and nonverbal interference on spatial and object visual working memory. Memory \& Cognition, 33, 203-212.

Proctor, R. W. (1983). Recognition memory for pictures as a function of poststimulus interval: An empirical clarification of existing literature. Journal of Experimental Psychology. Learning, Memory, and Cognition, 9, 256-262.

Reuter, B., \& Kathmann, N. (2004). Using saccade tasks as a tool to analyze executive dysfunctions in schizophrenia. Acta Psychologica, $115,255-269$.

Rosofsky, I., Levin, S., \& Holzman, P. S. (1982). Psychomotility in the functional psychoses. Journal of Abnormal Psychology, 91, $71-74$.

Sartori, E., \& Edan, G. (2006). Assessment of cognitive dysfunction in multiple sclerosis. Journal of the Neurological Sciences, 245, 169-175.
Simons, D. J. (1996). In sight, out of mind: When object representations fail. Psychological Science, 7, 301-305.

Sobin, C., \& Sackeim, H. A. (1997). Psychomotor symptoms of depression. The American Journal of Psychiatry, 154, 4-17.

Sperling, G. (1963). A model for visual memory tasks. Human Factors, 5, 19-31.

Swanson, H. L. (1999). What develops in working memory? A life span perspective. Developmental Psychology, 35, 986-1000.

Thomason, M. E., Race, E., Burrows, B., Whitfield-Gabrieli, S., Glover, G. H., \& Gabrieli, J. D. E. (2009). Development of spatial and verbal working memory capacity in the human brain. Journal of Cognitive Neuroscience, 21, 316-332.

Toepper, M., Gebhardt, H., Beblo, T., Thomas, C., Driessen, M., Bischoff, M., et al. (2010). Functional correlates of distractor suppression during spatial working memory encoding. Neuroscience, $165,1244-1253$

van der Wee, N. J., Ramsey, N. F., Jansma, J. M., Denys, D. A., van Megen, H. J., Westenberg, H. M., et al. (2003). Spatial working memory deficits in obsessive compulsive disorder are associated with excessive engagement of the medial frontal cortex. Neuroimage, 20, 2271-2280.

Visscher, K. M., Miezin, F. M., Kelly, J. E., Buckner, R. L., Donaldson, D. I., McAvoy, M. P., et al. (2003). Mixed blocked/ event-related designs separate transient and sustained activity in fMRI. Neuroimage, 19, 1694-1708.

Vogel, E. K., Woodman, G. F., \& Luck, S. J. (2006). The time course of consolidation in visual working memory. Journal of Experimental Psychology. Human Perception and Performance, 32, 1436-1451.

Vuontela, V., Rama, P., Raninen, A., Aronen, H. J., \& Carlson, S. (1999). Selective interference reveals dissociation between memory for location and colour. Neuroreport, 10, 2235-2240.

Wagenmakers, E.-J., \& Brown, S. (2007). On the linear relation between the mean and the standard deviation of a response time distribution. Psychological Review, 114, 830-841.

Wagenmakers, E.-J., Grasman, R. P. P. P., \& Molenaar, P. C. M (2005). On the relation between the mean and the variance of a diffusion model response time distribution. Journal of Mathematical Psychology, 49, 195-204.

Wright, A. A., Cook, R. G., Rivera, J. J., Shyan, M. R., Neiworth, J. J., \& Jitsumori, M. (1990). Naming, rehearsal, and interstimulus interval effects in memory processing. Journal of Experimental Psychology. Learning, Memory, and Cognition, 16, 1043-1059. 